\title{
MEROMORPHIC FUNCTIONS OF FINITE ORDER
}

\section{S. M. SHAH}

1. Introduction. Let $f(z)$ be an entire function of finite order $\rho$. If the genus $p$ of the canonical product (c.p.) formed with zeros of $f(z)$ is equal to the genus of $f(z)$, then it is known ${ }^{1}$ that $[9 ; 10]$

$$
\liminf _{r \rightarrow \infty} \frac{\log M(r)}{n(r, 0) \phi(r)}=0
$$

where $\phi(r)$ is any positive nondecreasing function such that

$$
\int_{1}^{\infty} d t / \phi(t)
$$

is convergent. Another proof of this theorem is given by Boas [2] where he assumes only that $\phi(r)$ is positive and (1) exists. Following the method of Boas, Shanker Hari has shown in a paper under publication that if $f(z)$ is a c.p. of order and genus $\rho$ then

$$
\liminf _{r \rightarrow \infty} \frac{\log M(r)}{N(r, 0) \phi(r)}=0 .
$$

In this paper we prove a more general theorem by the method given in $[9 ; 10]$.

Let $G, p_{1}, p_{2}$ denote respectively the genus of a meromorphic function $f(z)$, the genus of the c.p. $P_{1}$ formed with zeros of $f$ and the genus of the c.p. $P_{2}$ formed with poles of $f$. Write ${ }^{2} S=\max \left(p_{1}, p_{2}\right), n(t)$ $=n(t, f-0)+n(t, f-\infty), n_{1}(t)=n\left(t, P_{1}-0\right)+n\left(t, P_{2}-0\right)$. Let $k$ denote any constant $\geqq 1, \phi(x)$ any positive nondecreasing function such that (1) exists, $\left(a_{i}(z)\right), i=0,1, \cdots, l$ any meromorphic functions (including rational functions or finite constants) such that

$$
T\left(r, a_{i}(z)\right)=o(T(r, f)), \quad i=0,1, \cdots, l
$$

and $g_{0}(z)=g(z), g_{1}(z), g_{2}(z)$ any meromorphic functions (including rational functions or constants, finite or infinite) such that

$$
T\left(r, g_{i}(z)\right)=o(T(r, f)), \quad i=0,1,2 .
$$

Presented to the Society, November 29, 1958; received by the editors December 2, 1958.

${ }^{1}$ For notations see $7[7 ; 8]$.

2 The function $n(r, f-g)$ denotes the number of zeros of $f-g$ in $|z| \leqq r$. If $g$ is infinite constant, then $N(r, 1 /(f-g))=N(r, f)$. 
Write

$$
\psi(f)=\sum_{0}^{l} a_{i}(z) f^{(i)}(z), \quad \psi\left(f-g_{1}\right)=\sum_{0}^{l} a_{i}(z)\left\{f^{(i)}(z)-g_{1}{ }^{(i)}(z)\right\}
$$

when $g_{1}$ is not infinite constant; we suppose that $\left(a_{i}\right)$ are so chosen that neither $\psi(f)$ nor $\psi\left(f-g_{1}\right)$ reduces identically to a constant.

THEOREM 1. Let $f(z)$ be a meromorphic function of integer order $\rho$. (a) If $G=S$, then

$$
\begin{aligned}
& \liminf _{r \rightarrow \infty} \frac{T(k r, f)}{\{n(r, 0)+n(r, \infty)\} \phi(r)}=0, \\
& \liminf _{r \rightarrow \infty} \frac{T(k r, f)}{\{N(r, 0)+N(r, \infty)\} \phi(r)}=0 .
\end{aligned}
$$

(b) If $G>S$, then

$$
\bar{N}\left(r, \frac{1}{\psi(f)-1}\right) \sim T(r, f) \sim A r^{\rho} \quad(0<A<\infty) .
$$

THEOREM 2. Let $f(z)$ be a meromorphic function of noninteger order $\rho>0$. Then

(a)

$$
\liminf _{r \rightarrow \infty} \frac{T(k r, f)}{n\left(r, f-g_{1}\right)+n\left(r, f-g_{2}\right)} \leqq k^{1+S} B(S),
$$

(8) $\quad \liminf _{r \rightarrow \infty} \frac{T(k r, f)}{N\left(r, 1 /\left(f-g_{1}\right)\right)+N\left(r, 1 /\left(f-g_{2}\right)\right)} \leqq k^{1+S}(S+1) B(S)$ where

$$
\begin{aligned}
& S=[\rho], \quad A(S)=2 \\
& =2 e(2+\log S)(S+1)^{2} / S \\
& \text { if } S=0 \text {, } \\
& \text { if } S \geqq 1 \text {, }
\end{aligned}
$$

and

$$
B(S)=A(S) /(\rho-S)(1+S-\rho)
$$

and $g_{1}, g_{2}$ are any two distinct functions satisfying (3).

Corollary.

$$
\liminf _{r \rightarrow \infty} \frac{T(r, f)}{n(r, f-g)} \leqq 2 B(S)
$$




$$
\liminf _{r \rightarrow \infty} \frac{T(r, f)}{N(r, 1 /(f-g))} \leqq 2(S+1) B(S)
$$

except possibly for one $g$ in each relation (9), (10).

(b) If (10) does not hold for $g=g_{1}$, then for all $r>r_{0}$,

(11) $T(r, f)<\left\{\frac{2(S+1) B(S)}{2(S+1) B(S)-1}\right\}\left\{N(r, f)+\bar{N}\left(r, 1 /\left(\psi\left(f-g_{1}\right)-1\right)\right)\right\}$ if $g_{1} \not \equiv \infty$ and

(12) $T(r, f)<\left\{\frac{2(S+1) B(S)}{2(S+1) B(S)-1}\right\}\left\{N\left(r, \frac{1}{f}\right)+\bar{N}\left(r, \frac{1}{\psi(f)-1}\right)\right\}$ if $g_{1} \equiv \infty$.

(c) If (9) does not hold for $g=g_{1}$, then we have for a sequence of values of $r \uparrow \infty$,

$$
T(r, f)<\left\{\frac{2 \rho B(S)}{2 \rho B(S)-1}\right\}\left\{N(r, f)+\bar{N}\left(r, 1 /\left(\psi\left(f-g_{1}\right)-1\right)\right)\right\}
$$

if $g_{1} \not \equiv \infty$ and

$$
T(r, f)<\left\{\frac{2 \rho B(S)}{2 \rho B(S)-1}\right\}\{N(r, 1 / f)+\bar{N}(r, 1 /(\psi(f)-1))\}
$$

if $g_{1} \equiv \infty$.

THEOREM 3. Let $f(z)$ be an entire function of order $\rho$ and let, when $\rho>0, h(\rho)=\left(\rho+\left(1+\rho^{2}\right)^{1 / 2}\right)\left(1+\left(1+\rho^{2}\right)^{1 / 2}\right)^{\rho} / \rho^{\rho}$. If $\rho>0$, then

$$
\begin{aligned}
& \liminf _{r \rightarrow \infty} \frac{\log M(k r)}{n(r, f-g)} \leqq \frac{2 k^{\rho} h(\rho)}{\rho}, \\
& \liminf _{r \rightarrow \infty} \frac{\log M(k r)}{N(r, 1 /(f-g))} \leqq 2 k^{\rho} h(\rho)
\end{aligned}
$$

for every entire function $g(z)$ (including a polynomial or a finite constant) satisfying (3), with one possible exception. If $\rho=0$, then

$$
\liminf _{r \rightarrow \infty} \frac{\log M(k r)}{N(r, 1 /(f-g))}=1
$$

for every entire function $g(z)$ such that $M(r, g)=o(M(r, f))$.

THEOREM 4. Let $f(z)$ be a meromorphic function of order $\rho$. If $\rho>0$, then 


$$
\begin{aligned}
& \liminf _{r \rightarrow \infty} \frac{T(k r, f)}{n(r, f-g)} \leqq \frac{3 k^{\rho}}{\rho}, \\
& \liminf _{r \rightarrow \infty} \frac{T(k r, f)}{N(r, 1 /(f-g))} \leqq 3 k^{\rho}
\end{aligned}
$$

for every meromorphic function $g(z)$ satisfying (3), with two possible exceptions. If $\rho=0$ then

$$
\liminf _{r \rightarrow \infty} \frac{T(k r, f)}{N\left(r, 1 /\left(f-g_{1}\right)\right)+N\left(r, 1 /\left(f-g_{2}\right)\right)} \leqq 1
$$

where $g_{1}, g_{2}$ are any two distinct functions satisfying (3).

2. Remarks. (i) Let $\delta(\alpha)$ denote Nevanlinna defect. If $\sum \delta(\alpha)<2$, then $G=S$ and (4) holds. If $\sum \delta(\alpha)=2$, then (4) may or may not hold. (See [13, pp. 590-591] for an entire function of which the genus is greater than the genus of its c.p. and for which $\sum \delta(\alpha)=2$ and (4) holds.) Conversely if (4) holds, then $\sum \delta(\alpha)$ may have its maximum value 2 or $\sum \delta(\alpha)<2(f(z)=\sin z)$. If (4) does not hold then $G>S$, $\delta(0)=\delta(\infty)=1$.

(ii) For entire functions of order $\rho, 0 \leqq \rho<1$, it is known that $[1 ; 11]$

$$
\liminf _{r \rightarrow \infty} \frac{\log M(r)}{N(r, a)} \leqq \frac{1}{1-\rho}
$$

and for meromorphic functions of order $\rho, 0 \leqq \rho<1$, it is known that

$$
\liminf _{r \rightarrow \infty} \frac{T(r)}{N(r, a)+N(r, b)} \leqq \frac{1}{1-\rho} .
$$

More precise results in this direction have been given by Edrei and Fuchs [4].

(iii) For entire functions of noninteger order $\rho>0$, it is known that

$$
\mathrm{L}(f, a)=\liminf _{r \rightarrow \infty} \frac{\log M(r)}{n(r, f-a)}<\infty
$$

for every finite $a$. A sharp upper bound for $L(f, a)$ when $\rho>1$, is not known but by (15) $L(f, a) \leqq 2 h(\rho) / \rho$ except possibly for one value of $a$. This exceptional value may exist. Consider [7, pp. 18-19]

$$
f(z)=\prod_{2}^{\infty} E\left(\frac{z}{a_{n}}, p\right), \quad a_{n}=-n^{1 / \rho}, \quad p<\rho<p+1 .
$$

$f(z)$ is an entire function of order $\rho$ and 


$$
\log M(r) / n(r, 0) \sim \pi / \operatorname{Sin} \pi(\rho-p)
$$

which can be made greater than $2 h(\rho) / \rho$ by choosing $\rho-p$ sufficiently small.

(iv) If (15), (16), (18) or (19) do not hold for $g=g_{1}$ then we can obtain relations similar to (11)-(14). For instance if (15) or (16) does not hold when $g=g_{1}$, then

$$
\liminf _{r \rightarrow \infty} \frac{T(r, f)}{\bar{N}\left(r, 1 /\left(\psi\left(f-g_{1}\right)-1\right)\right)} \leqq 2 .
$$

\section{Theorem 1.}

Lemma 1. If $f(z)=z^{v} \exp (Q(z)) P_{1}(z) / P_{2}(z)$ is a proper meromorphic function $^{3}$ of order $\rho \geqq 0$ such that $G=S$, then for all $r>r_{0}$

$$
T(k r, f) \leqq A(S) k^{1+S} J(r, S)
$$

where $e^{4}$

$$
\begin{aligned}
A(S) & =2 & & \text { if } S=0, \\
& =2 e(2+\log S)(S+1)^{2} / S & & \text { if } S \geqq 1,
\end{aligned}
$$

and

$$
J(r, S)=r^{1+S} \int_{0}^{\infty} \frac{n_{1}(t) d t}{t^{1+S}(t+r)} .
$$

Proof. We have

$$
T(k r, f)=O\left(r^{S}+\log r\right)+\log M\left(k r, P_{1}\right)+\log M\left(k r, P_{2}\right)
$$

and when $p_{1} \geqq 1[8$, pp. 225-226]

$\log M\left(k r, P_{1}\right)$

$$
\leqq\left(2+1 / p_{1}\right) e\left(2+\log p_{1}\right)\left(1+p_{1}\right) k^{1+p 1} r^{1+p 1} \int_{0}^{\infty} \frac{n\left(t, P_{1}-0\right) d t}{t^{1+p 1}(t+k r)} .
$$

Hence, when $p_{1}=p_{2} \geqq 1$,

$$
T(k r, f) \leqq \frac{2 e(2+\log S)(1+S)^{2}}{S} k^{1+S} J(r, S) .
$$

If $p_{1}=p_{2}=0$, then

$$
T(k r, f)<O(\log r)+k r \int_{0}^{\infty} \frac{n_{1}(t) d t}{t(t+k r)} .
$$

${ }^{8} f$ is not a rational function.

We can take $A(0)=1+c$, where $c$ is any positive number. 
If $p_{1}>p_{2}$ then $\log M\left(k r, P_{2}\right)=o\left(r^{1+p_{2}}\right)=o\left(r^{p_{1}}\right)$ and a similar result if $p_{1}<p_{2}$. Hence the lemma is proved.

LEMMA 2. If $f(z)$ is a proper meromorphic function of finite order and

$$
\psi(f)=\psi(z)=\sum_{0}^{l} a_{i}(z) f^{(i)}(z)
$$

then

$$
(1+o(1)) T(r, f)<\bar{N}(r, f)+\bar{N}\left(r, \frac{1}{\psi(f)-1}\right)+N\left(r, \frac{1}{f}\right) .
$$

This lemma is substantially contained in Theorem 7 of [5].

Lemma 3. If $f(z)=z^{v} \exp (Q(z)) P_{1}(z) / P_{2}(z)$ and $G>S$ then $Q(z)$ $=a_{\rho} z^{\rho}+\cdots,\left|a_{\rho}\right|=A \neq 0$ and

(i) $T(r, f) \sim A r^{p}$,

(ii) $T\left(r, f^{\prime}\right) \sim A r^{p}$,

(iii) $T(r, \psi)=T\left(r, \sum_{0}^{\prime} a_{i} f^{(i)}\right) \sim A r^{p}$.

Proof. Write $f=b_{0} \exp (Q)$ where $b_{0}=z^{v} P_{1} P_{2}^{-1}$,

$$
\begin{aligned}
T(r, f) & \leqq T(r, \exp Q)+T\left(r, b_{0}\right), \\
T(r, \exp Q) & \leqq T(r, f)+T\left(r, b_{0}\right)+O(1)
\end{aligned}
$$

and since $T(r, \exp Q) \sim A r^{\rho}, T\left(r, b_{0}\right)=o\left(r^{\rho}\right)$, (i) follows.

$$
\begin{aligned}
f^{\prime} & =e^{Q}\left\{k z^{k-1} P_{1} P_{2}^{-1}+z^{k} Q^{\prime} P_{1} P_{2}^{-1}+z^{k} P_{1}^{\prime} P_{2}^{-1}-Z^{k} P_{1} P_{2}^{-2} P_{2}^{\prime}\right\} \\
& =e^{Q} b_{1} \text { (say). }
\end{aligned}
$$

Then $T\left(r, b_{1}\right)=o\left(r^{\rho}\right)$ and so $T\left(r, f^{\prime}\right) \sim A r^{\rho}$.

(iii) $f^{\prime \prime}=(\exp Q) b_{2}$ where $T\left(r, b_{2}\right)=o\left(r^{p}\right)$ and so on. Hence

$$
\begin{aligned}
T(r, \psi(f)) & =T\left(r,(\exp Q) \sum a_{i} b_{i}\right) \\
& \leqq T(r, \exp Q)+T\left(r, \sum a_{i} b_{i}\right) \\
& \leqq(A+o(1)) r^{\rho}+\sum T\left(r, a_{i}\right)+\sum T\left(r, b_{i}\right) \\
& =(A+o(1)) r^{\rho} .
\end{aligned}
$$

Also

$$
\exp Q=\psi(f) / \sum a_{i} b_{i}, \quad T(r, \exp Q) \leqq T(r, \psi(f))+o\left(r^{\rho}\right)
$$

and hence $T(r, \psi(f)) \sim A r^{\rho}$.

(a) Proof $\mathrm{OF}^{5}(4)$. Since $\rho \geqq 1$ and $G=S, n(x)$ tends to infinity with

b (4) can also be proved by the method given in [12, pp. 696-697]. 
$x$. Further $T(k r, f) \sim T\left(k r, f z^{v}\right)$ where $v$ is any integer and so we may suppose $f(0) \neq 0, \neq \infty$. Then $f(z)=\exp (Q(z)) P_{1}(z) / P_{2}(z)$. Since $G=S$, we have $S=\rho$ or $\rho-1$. Consider first when $S=\rho$. If $n(x)=O\left(x^{S}\right)$ we have

$$
J(r, S)<c_{1} r^{S} \int_{0}^{r} \frac{n(x) d x}{x^{1+S}}
$$

and hence from Lemma 1 of $[9$, p. 24] we get

$$
\liminf _{r \rightarrow \infty} J(r, S) / n(r) \phi(r)=0 .
$$

If $\lim \sup _{x \rightarrow \infty} n(x) / x^{S}=\infty$, we write $y=\log x$ and $\log \left(n(x) / x^{S}\right)$ $=\log \mu(x)=\psi(y)$. Then lim $\sup _{y \rightarrow \infty} \psi(y)=\infty$, and since $f(z)$ is of order $\rho=S, \lim \sup _{y \rightarrow \infty} \psi(y) / y=0$. Apply Lemma 2 of $[9$, p. 25]. There exists a sequence $r_{n} \uparrow \infty$ for which

$$
\begin{array}{rlrl}
\mu(x) & <\mu\left(r_{n}\right), & \Delta<x & <r_{n}, \\
\frac{\log \mu(x)}{\log x} & <\frac{\log \mu\left(r_{n}\right)}{\log r_{n}}, & x>r_{n} .
\end{array}
$$

Hence

$$
J\left(r_{n}, S\right)<c_{2} n\left(r_{n}\right) \log r_{n}
$$

Consider now $S=\rho-1$. Given $\phi(x)$ we can find [10] a function $\phi_{1}(x)$ such that for all large $x$ : (i) $\phi_{1}(x) \leqq \phi(x)$; (ii) $\phi_{1}(x) / x^{\alpha}$ is nonincreasing, $\alpha$ being a positive number less than unity; (iii) $\int_{1}^{\infty} d t / t \phi_{1}(t)$ is convergent. If now

$$
\limsup _{x \rightarrow \infty} n(x) \phi_{1}(x) / x^{1+S}>0,
$$

then for a sequence $R_{n} \uparrow \infty, J\left(R_{n}, S\right)=o\left(n\left(R_{n}\right) \phi_{1}\left(R_{n}\right)\right)$. If $n(x) \phi_{1}(x)$ $=o\left(x^{1+S}\right)$ then we choose in Lemma 3 of $[10$, p. 184] $0<\beta<1-\alpha$, $\theta(x)=x^{\beta}, \Psi(x)=n(x) \phi_{1}(x) / x^{S+1-\beta}$ and obtain for $x_{n} \uparrow \infty, J\left(x_{n}, S\right)$ $=o\left(\phi\left(x_{n}\right) n\left(x_{n}\right)\right)$ and (2) is proved.

Since $n(r, a) \leqq N(2 r, a) / \log 2$, (5) follows from (4).

(b) Proof of (6). By hypothesis

$$
N(r, f)=o\left(r^{p}\right), \quad N(r, 1 / f)=o\left(r^{p}\right),
$$

and so from (21)

$$
(1+o(1)) T(r, f)<\bar{N}(r, 1 /(\psi(f)-1))<T(r, \psi(f))+O(1)
$$

and so (6) follows from Lemma 3(i) and (iii). 
4. Theorems $2,3,4$.

LEMMA 4. If $f(z)$ is a meromorphic function of order $\rho>0$, then

$$
I_{1}(r)=\int_{1}^{r} \frac{T(k t, f)}{t} d t<\frac{k^{\rho}}{\rho}(1+\epsilon) T(r, f)
$$

for a sequence of values of $r$ tending to infinity.

LEMma 5. If $f(z)$ is an entire function of order $\rho>0$, then

$$
I_{2}(r)=\int_{1}^{r} \frac{\log M(k t)}{t} d t<\frac{k^{\rho}}{\rho} h(\rho)(1+\epsilon) T(r, f)
$$

where $h(\rho)$ has been defined in the statement of Theorem 3, for a sequence of values of $r$ tending to infinity.

The proof of Lemma 4 is straightforward (cf. [14, p. 321]) and the proof of Lemma 5 depends on the relation

$$
\log M(r) \leqq \frac{h+1}{h-1} T(h r),
$$

We obtain for a sequence of values of $r \rightarrow \infty$,

$$
I_{2}(r) \leqq \frac{h+1}{h-1} \frac{h^{\rho} k^{\rho}}{\rho}(1+\epsilon) T(r) .
$$

Choose $h=\left(1+\left(1+\rho^{2}\right)^{1 / 2}\right) / \rho$.

(a) Proof of (7). We have $S=[\rho]$

$$
T(k r, f)<A(S) k^{1+S} J(r, S),
$$$$
r>r_{0}
$$

We choose in Lemma 3 of $[10$, p. 184] (see also [12, p. 69]) $\Psi(x)$ $=n_{1}(x) / x^{p-\epsilon}$ and obtain that for $r=r_{n} \uparrow \infty$

$$
T\left(k r_{n}, f\right)<B(S)\left(1+\epsilon^{\prime}\right) k^{1+S} n\left(r_{n}\right) .
$$

Let $H(z)=\left(f(z)-g_{1}(z)\right) /\left(f(z)-g_{2}(z)\right), g_{1} \not \equiv \infty, g_{2} \not \equiv \infty$. Then $T(r, H)$ $\sim T(r, f)$. Also for a sequence $R_{n} \uparrow \infty$,

$$
T\left(k R_{n}, H\right)<B(S)\left(1+\epsilon^{\prime}\right) k^{1+S}\left\{n\left(R_{n}, H-0\right)+n\left(R_{n}, H-\infty\right)\right\} .
$$

Now

$$
n\left(R_{n}, H-0\right)+n\left(R_{n}, H-\infty\right)<\sum_{1}^{2} n\left(R_{n}, f-g_{i}\right)+\sum_{1}^{2} n\left(R_{n}, g_{i}-\infty\right) \text {. }
$$

Suppose now $k>1$. Then

$$
n\left(R_{n}, g_{i}-\infty\right)<N\left(k R_{n}, g_{i}\right) / \log k=o\left(T\left(k R_{n}, f\right)\right) .
$$


Hence for $n>n_{1}$

$$
T\left(k R_{n}, f\right)<B(S)\left(1+\epsilon^{\prime}\right) k^{1+S} \sum_{1}^{2} n\left(R_{n}, f-g_{i}\right) .
$$

If we take $H(z)=f(z)-g_{2}(z), g_{2} \not \equiv \infty$, we get for another $\left\{R_{n}\right\} \uparrow \infty$, (23) $T\left(k R_{n}, f\right)<B(S)\left(1+\epsilon^{\prime}\right) k^{1+S}\left\{n\left(R_{n}, f-\infty\right)+n\left(R_{n}, f-g_{2}\right)\right\}$ and so from (22) and (23)

$$
\liminf _{r \rightarrow \infty} T(k r, f) / \sum_{1}^{2} n\left(r, f-g_{i}\right) \leqq B(S) k^{1+S}
$$

and ( 7$)$ is proved when $k>1$. Further $k$ can be chosen arbitrarily near to 1 in the relation

$$
\liminf _{r \rightarrow \infty} T(r, f) / \sum_{1}^{2} n\left(r, f-g_{i}\right) \leqq B(S) k^{1+S}
$$

and so (7) is true when $k \geqq 1$.

Proof of (8). Write

$$
N_{1}(t)=\int_{0}^{r}\left\{n_{1}(t) / t\right\} d t ; \quad N(t)=N(t, f)+N(t, 1 / f) .
$$

Then

$$
\begin{aligned}
J(r, S) & =\int_{0}^{\infty} \frac{r^{1+S} n_{1}(t) d t}{t^{1+S}(t+r)} \\
& <(S+1)\left\{r^{S} \int_{0}^{r} \frac{N_{1}(t) d t}{t^{1+S}}+r^{1+S} \int_{r}^{\infty} \frac{N_{1}(t) d t}{t^{2+S}}\right\} \\
& <(S+1)(1+\epsilon)\left\{r^{S} \int_{x_{0}}^{r} \frac{[N(t)] d t}{t^{1+S}}+r^{1+S} \int_{r}^{\infty} \frac{[N(t)] d t}{t^{S+2}}\right\}
\end{aligned}
$$

if $r>x_{1}(\epsilon)$. Hence by Lemma 3 of $[10$, p. 184] we get

$$
\liminf _{\boldsymbol{r} \rightarrow \infty} \frac{T(k r, f)}{N(\boldsymbol{r})} \leqq B(S)(1+S) k^{1+S}
$$

and the rest of the argument is the same as for (7). Hence (8) is proved. The Corollary follows from (7) and (8).

(b) If (10) is false for $g=g_{1} \neq \infty$, then

$$
\liminf _{r \rightarrow \infty} \frac{T(r, f)}{N\left(r, 1 /\left(f-g_{1}\right)\right)}=\beta>2(S+1) B(S) .
$$


Hence for all $r>r_{0}(\epsilon)$,

$$
T(r, f)>(\beta-\epsilon) N\left(r, 1 /\left(f-g_{1}\right)\right)
$$

We apply Lemma 2 to $f-g_{1}$ and obtain

$$
\begin{aligned}
& \{1+o(1)\} T\left(r, f-g_{1}\right) \\
& \quad \leqq \bar{N}\left(r, f-g_{1}\right)+\bar{N}\left(r, \frac{1}{\psi\left(f-g_{1}\right)-1}\right)+N\left(r, \frac{1}{f-g_{1}}\right),
\end{aligned}
$$

and for $r>r_{1}(\epsilon)$

$$
\left(1-\frac{1}{\beta-\epsilon}-\epsilon\right) T(r, f) \leqq N(r, f)+\bar{N}\left(r, \frac{1}{\psi\left(f-g_{1}\right)-1}\right) .
$$

Hence for $r>r_{2}$

$$
T(r, f)<\left\{\frac{2(S+1) B(S)}{2(S+1) B(S)-1}\right\}\left\{N(r, f)+\bar{N}\left(r, \frac{1}{\psi\left(f-g_{1}\right)-1}\right)\right\} .
$$

The proof of (12) is similar.

(c) We omit the proofs of (13)-(14) which can be proved with the help of Lemma 4.

(d) The first part of Theorem 3 follows from Lemma 5 and (17) follows from a result of Boas $[1$, pp. $6-7 ; 3$, p. 48]. We omit the proofs of Theorems 3 and 4 .

5. Example. We show that (4), (5) are best possible in the sense that $k$ cannot be replaced by a function $\alpha(r)$ tending to infinity with $r$. We prove that given $\alpha(r) \rightarrow \infty$ with $r$, there is a function $\phi(x)$ such that (1) exists, and an entire function (c.p.) $f(z)$ of integer order $\rho$ and genus $\rho-1$ such that

(25) $\liminf _{r \rightarrow \infty} \frac{T(r \alpha(r), f)}{N(r, 0) \phi(r)}=\liminf _{r \rightarrow \infty} \frac{T(r \alpha(r), f)}{n(r, 0) \phi(r)} \geqq \frac{1}{\left(\left(1+2^{1 / 2}\right)^{2}\right.} \quad$ if $\rho=1$

$$
=\infty \quad \text { if } \rho>1 \text {. }
$$

(a) We suppose that $\alpha(r)$ satisfies the following:

(i) $\alpha(r)>0$ for $r \geqq x_{0}>0$ and tends to infinity with $r$.

(ii) $\alpha^{\prime}(r)>0$ and $r \alpha^{\prime}(r) \downarrow$ for $r \geqq x_{0}$.

(iii) $r^{2} \alpha^{\prime}(r) / \alpha^{2}(r)$ is strictly increasing for $r \geqq x_{0}$. For instance we can take $\alpha(r)=l_{k} r, k=1,2, \cdots,\left(l_{1} r=\log r ; l_{k}(r)=\log \left(l_{k-1} r\right)\right)$. A function of slower growth and satisfying (i)-(iii) is given by the functional equation $\alpha\left(e^{x}\right)=e \alpha(x)$. Let

$$
\begin{aligned}
\phi(x) & =\alpha^{2}(x) / x \alpha^{\prime}(x) & x \geqq x_{0}, \\
& =\phi\left(x_{0}\right) & 0 \leqq x<x_{0},
\end{aligned}
$$




$$
\begin{aligned}
n(x, 0) & =[x / \phi(x)] \\
& =0
\end{aligned}
$$$$
x \geqq x_{0},
$$$$
0 \leqq x<x_{0} \text {. }
$$

From (ii), (iii) it is seen that $\phi(x)$ and $x / \phi(x) \uparrow$ with $x$. Further

$$
\int_{0}^{x} \frac{n(t, 0)}{t^{2}} d t<\int_{x_{0}}^{x} \frac{d t}{t \phi(t)} \rightarrow \frac{1}{\alpha\left(x_{0}\right)}
$$

as $X \rightarrow \infty$. Hence (1) is convergent. Write $R=r \alpha(r)$. Then

$$
\alpha(R)-\alpha(r)=\int_{r}^{R} \alpha^{\prime}(t) d t=o(\alpha(R)) .
$$

Hence $\alpha(R) \sim \alpha(r)$ and so $\alpha(r)$ is "slowly increasing" function [6] and

$$
\alpha(r)=o\left(r^{\delta}\right), \quad \delta>0, \quad \int_{x_{0}}^{r} \frac{d t}{\alpha(t)} \sim \frac{r}{\alpha(r)} .
$$

From (ii) we have $\alpha(r)=o(\phi(r))$. Let

$$
f(z)=\prod_{1}^{\infty}\left(1+\frac{z}{r_{n}}\right)
$$

where $r_{n}>0, n=1,2, \cdots$, and are given by $n(x, 0)$. (The first few zeros may be equal; cf. [8, p. 228].) From (26) we see that the series $\sum 1 / r_{n}$ is convergent and $f(z)$ is an entire function (c.p.) of genus $p=0$. Now

$$
\begin{aligned}
\log M(r) & <r \int_{0}^{r} \frac{n(t, 0)}{t} d t+r \int_{r}^{\infty} \frac{n(t, 0)}{t^{2}} d t \leqq\{1+o(1)\} \frac{r}{\alpha(r)}, \\
\log M(r) & >r \int_{R}^{\infty} \frac{n(t, 0) d t}{t(t+r)} \\
& >r \int_{R}^{\infty}\left\{\frac{t}{\phi(t)}-1\right\} \frac{d t}{t(t+r)}=\frac{r}{\alpha(r)}\{1+o(1)\} .
\end{aligned}
$$

Hence

$\log M(r) \sim r / \alpha(r), \log M(R) \sim r, n(r, 0) \phi(r) \sim r \sim N(r, 0) \phi(r)$,

$$
\begin{aligned}
T(R) & \geqq \frac{\beta-1}{\beta+1} \log M\left(\frac{R}{\beta}\right), \\
& \sim \frac{\beta-1}{\beta+1} \frac{R}{\beta \alpha(r)}=\frac{\beta-1}{\beta+1} \frac{r}{\beta} .
\end{aligned}
$$

Choose $\beta=1+2^{1 / 2}$ and (25) follows when $\rho=1$. 
(b) If $\rho>1$ then let

$$
f(z)=\prod_{1}^{\infty}\left\{1+\frac{z^{\rho}}{r_{n}}\right\}
$$

where $r_{n}$ have been defined in (a). $f(z)$ is an entire function (c.p.) of order $\rho$ and genus $\rho-1$. We have for all $r>r_{0}$

$$
\begin{aligned}
\log M(k r, f) & <c_{3} n(r, f-0) \phi(r) / \alpha(r), \\
\log M(R, f) & \geqq \frac{1}{\rho}(1+o(1))\left(\alpha(r)^{\rho-1} n(r, f-0) \phi(r),\right. \\
T(R, f) & >c_{4}(\alpha(r))^{\rho-1} n(r, f-0) \phi(r), \\
T(R, f) & >c_{4}(\alpha(r))^{\rho-1} N(r, 1 / f) \phi(r)
\end{aligned}
$$

and (25) is proved for $\rho>1$.

\section{REFERENCES}

1. R. P. Boas, Rend. Circ. Mat. Palermo vol. 1 (1952) pp. 1-9.

2. - J. London Math. Soc. vol. 28 (1953) pp. 194-196.

3. - Entire functions, New York, Academic Press, 1954.

4. A. Edrei and W. H. J. Fuchs, Abstract 546-63, Notices Amer. Math. Soc. vol. 6 (1958) p. 371.

5. W. K. Hayman, Rend. Circ. Mat. Palermo vol. 2 (1953) pp. 346-392.

6. G. H. Hardy and W. W. Rogosinki, Quart. J. Math. Oxford Ser. (2) vol. 16 (1945) pp. 49-58.

7. R. Nevanlinna, Le theoreme de Picard-Borel et la theorie des fonctions meromorphes, Paris, 1929.

8. - Eindeutige Analytische Funktionen, Berlin, 1953.

9. S. M. Shah, J. London Math. Soc. vol. 15 (1940) pp. 23-31.

10. — J. Indian Math. Soc. vol. 5 (1941) pp. 179-188.

11. —- Math. Student vol. 12 (1944) pp. 67-70.

12. —- Proc. Amer. Math. Soc. vol. 2 (1951) pp. 694-698.

13. — , Duke Math. J. vol. 19 (1952) pp. 585-593.

14. — J. Indian. Math. Soc. vol. 20 (1956) pp. 315-327.

UNIVERSITY OF WisCONSIN AND Muslim University, Aligarh, India 